\title{
Hydatid Disease and Pregnancy: A Short Note
}

\author{
Maria A Grácio* and António J Santos Grácio \\ Institute of Hygiene and Tropical Medicine / New University of Lisbon, Lisbon, Portugal
}

${ }^{\star}$ Corresponding Author: Prof. Maria A Grácio, Institute of Hygiene and Tropical Medicine / New University of Lisbon, Rua da Junqueira 100, 1348-008 Lisbon, Portugal; Email: mameliahelm@ihmt.unl.pt

Received: May 24, 2019; Accepted: June 13, 2019; Published: July 06, 2019;

\section{Short Commentary}

Tapeworm is the common name of the cestode worm, Echinococcus granulosus, which is the causal agent of hydatid disease in humans. This helminth infection is also known as cystic echinococcosis and hydatidosis, and is included in the zoonoses group. According to WHO [1]: (i) more than 1 million people are affected with echinococcosis at any one time; (ii) infection is globally distributed and found in every continent except Antarctica; (iii) robust surveillance is fundamental in order to show burden of disease and to evaluate progress and success of control programmes. However, as for other neglected diseases which are focused in underserved populations and remote areas, data is especially scarce and will need more attention if control programmes are to be implemented and measured; (iv) ultrasonography imaging is the technique of choice for diagnosis of cystic echinococcosis; (v) cysts can be incidentally discovered by radiography. Specific antibodies are detected by different serological tests and can support the diagnosis. Biopsies and ultrasound-guided punctures may also be performed for differential diagnosis of cysts from tumors and abscesses; (vi) cystic echinococcosis are often expensive and completed to treat, sometimes requiring extensive surgery and/or prolonged drug therapy.

As to transmission: (i) a number of herbivorous and omnivorous animals act as intermediate hosts of E. granulosus. They become infected by ingesting the parasite eggs in contaminated food and water, and the parasite then develops into larval stages in the viscera; (ii) carnivores act as definitive hosts for the parasite, and host the mature tapeworm in their intestine. They are infected through the consumption of viscera of intermediate hosts that harbor the parasite; (iii) humans act as so- called accidental intermediate hosts in the sense that they acquire infection in the same way as other intermediate hosts, but are not involved in transmitting the infection to the definitive host; (iv) several distinct genotypes of E. granulosus are recognized some having distinct intermediate hosts preferences. Some genotypes are considered species distinct from E. granulosus. Not all genotypes cause infections in humans. The genotype causing the great majority of cystic echinococcosis infections in humans is principally maintened in a dog-sheep-dog cycle, yet several other domestic animals may also be involved, including goats, swine, cattle, camels and yaks; (v) human infection with E. granulosus leads to the development of one or more hydatid cysts located most often in the liver and lungs, and less frequently in the bones, kidney, spleen, muscles, central nervous system and eyes; (vi) abdominal pain, nauseas and vomiting are commonly seen when hydatids occur in the liver. If the lung is affected, clinical signs include chronic cough, chest pain and shortness of breath. Other signs depend on the location of the hydatid cysts and the pressure exerted on the surrounding tissues.

These results of the parasitism for tapeworms in humans show that it may have direct and indirect consequences in human health, in general. cause devastating morbidity with severe consequences in general and in female reproductive health. Here our objective is to alert medical practitioners of gynecology and obstetrics for hydatid cyst in pregnancy.

The authors: (2) report that "Incidence of hydatid disease in pregnancy ranges from 1 in 20,000 to 1 in 30,000. The diagnosis of liver hydatid cyst is not difficult but management during pregnancy is problematic. Both medical and surgical treatments are available but there is no consensus and each case has to be individualized"; (3) report "we present a 32-years old multigravida at 25 weeks of pregnancy in whom splenic and liver cysts were diagnosed by ultrasonography and magnetic resonance imaging (MRI). The splenic cyst was removed and a healthy baby was delivered vaginally at term"; (4) report "Case report A 25-year-old, gravida 1, para 0 pregnant female with history of liver cyst was admitted to the obstetric outpatient clinic for checkup. Patient who did not have any complaints was redirected to the radiology clinic for a second trimester ultrasound scan. Abdominal ultrasound revealed lesions of type-1, hydatid cyst $84 \times 67 \mathrm{~mm}$ in size at the right lobe fifth segment, and $67 \times 64 \mathrm{~mm}$ in size at the seventh segment of the liver, with millimetric echogenic female cysts and welldefined thickwalls. In addition, a type- 2 hydatid cyst with membrane dissociation, $55 \times 52 \mathrm{~mm}$ in size, was observed at the caudate lobe. An intrauterine fetus with fetal heart rate was observed with a bipariental diameter concordant with 21 weeks of gestation. Besides, there was a cystic lesion with fine septations surrounding the uterus. The patient was admitted to the obstetric care service with hydatid cyst hydatidosis in the vicinity of the uterus; (5) report "A young, apparently healthy from a rural area in South Africa presented in the third trimester of pregnancy with a symptomatic abdominal mass between the uterine fundus and liver. The etiology was established to be an echinococcus cyst of the liver and medical treatment was initiated. The fetal outcome was good but the mother died 3 days postpartum due to an unusual but devastating complication of the hydatid cyst; (6) report the management of hydatid disease in pregnancy, and they write that "in this review, we have attempted to summarize the presentation 
and available management approaches to hydatid disease, and have suggested evidence-based guidelines for its management during pregnancy; (7) report 2 cases of hydatid disease of liver during pregnancy, and they also made a review of literature; (8) report "A huge primary hydatid cyst of uterus" and they made also a review of literature.

Final conclusion: 1 - we think that it was here demonstrated the importance of the hydatid cyst as an underlying cause of negative effects in pregnancy; 2 - surgery in patients with hydatid cysts needs special care before, during or after surgery. Effectively, opening of a hydatid cyst requires special care not to spill the contents into the peritoneal cavity or tissues, since this may result in an anaphylactic reaction to the spilt fluid or dissemination and implantation of the immature scolices contained in the "sand" in the fluid. Incomplete removal of viable germinal epithelium from the liming of a hydatid cyst results in the formation of multiple cysts: 3 - we are in agreement with the conclusions of the authors (9): E. granulosus can affect any organ in the body from head to toe, and high suspicion of this disease is justified in endemic regions.

Keywords: Helminths; Cestodes; Tapeworms; Echinococcus granulosus; Hydatid Disease; Pregnancy; Gynecology; Obstetrics.

\section{References}

1. WHO (18 February 2018) Echinococcosis Key facts.

2. Ghosh JK, Goyal SK, Behera MK, Dixit VK, Jain AK. 2014 Hydatid cyst of liver presented as obstructive jaundice in pregnancy; Managed by PAIR. Journal of Clinical and Experimental Hepatology, https://doi.org/10.1016/j.jceh.2014.11.002

3. Can D, Oztekin O, Oztekin O, Tinar S, Sanci M. Hepatic and splenic hydatid cyst during pregnancy: a case report. Archives of Gynecology and Obstetrics, August 2003, 268(3): 239-240.

4. Tekin AF, Yilmaz H, Kara T, Seçkin E, Aybay MN, Alkan E, Case report A very rare case hydaid bcyst surrounding uterus and magnetic resonance imaging findings in the pregnant patient, 2018 Available online at www.sciencedirect.com.

5. Robertson M, Geerts L, Gebhardt S. A case of hidatid cyst associated with postpartum maternal dead. Ultrasound Obstet Gynecol 2006; 27: 693-696.

6. Rodrigues G, Seetharam P. Management of hydatid disease (echinococcosis) in pregnancy. Obstet Gynecol Surv, 2008 Feb; 63 (2): 116-123.

7. Demirel E, Ekmekci E, Izmirkatip C., Keeckci S, Gencdal S. Hydatid disease of liver during pregnancy: Report of two cases and Review of literature. 2018, Jacobspublishers.com, email: erem.dr@hotmail.com

8. Slimane NN, Taieb M, Khiali R, Rabehi H, Bekhouuche R, et al. A huge primary hydatid cyst of uterus. A case report and review of literature. J Univer Surg 2018; 6(2): 12 (5pags)

9. Sachar S, Goyal S, Sangwan S. Uncommon locations and presentations of hydatid cyst. Ann Med Health Sci Res 2014; 4: 447-452.

Citation:

Maria A Grácio and António J Santos Grácio (2019) Hydatid Disease and Pregnancy: A Short Note. Integr Gyn Obstet J Volume 2(3): 1-2. 